\title{
Gestaltungsmöglichkeiten für online Unterricht bei der Montage biegeweicher Materialien
}

\author{
Y. Kyosev \\ ${ }^{1}$ Professur Entwicklung und Montage von textilen Produkte, Institut für Textilmaschinen und Textile Hochleistungswerkstofftech- \\ nik (ITM), Fakultät Maschinenwesen, TU Dresden
}

\begin{abstract}
In diesem Beitrag wird ein Erfahrungsbericht über die Umsetzung der Online-Lehre und eine konkrete Aufgabenstellung während des COVID-Semesters in den Jahren 2020 und 2021 vorgestellt. Die Montage von flexiblen Materialien beinhaltet viele manuelle Tätigkeiten, die bisher von den Studierenden während der Praktika selbst durchgeführt werden konnten. Gezeigt werden zwei Aufgaben zur Online-Unterstützung der Lehre durch die Programmierung einer 3D-Visualisierung der Stichtypen und der Dokumentation der Montagevorgänge durch Video oder Bildund Textreihen. Die Erfahrungen zeigten, dass diese Visualisierungsvarianten den Personalaufwand etwas reduzieren können, aber das Mitführen eines Abspielgerätes (Tablet) von jedem Studenten während des Praktikums erfordern. Die Erfahrungen aus den beiden Aufgaben sollen auch für die Zukunft genutzt werden, um den Studenten mehr eigenständige Aufgaben anzubieten.

This paper presents an experience report on the implementation of online teaching and a concrete task during the COVID semester in 2020 and 2021. The assembly of flexible materials involves many manual operations, which until now could be performed by the students themselves during the practical courses. Shown are two tasks for online support of teaching by programming 3D visualisation of stitch types and documentation of assembly operations by video or series of images and texts. The experience showed that these visualisation variants can reduce the staff input a little, but require carrying a playback device (tablet) from each student during the practical. The experiences from the two tasks will also be used for the future to offer the students more independent tasks.
\end{abstract}

*Corresponding author: yordan.kyosev@tu-dresden.de 


\section{Einführung}

In der Veranstaltung "Maschinen und Verfahren der Konfektionstechnik" lernen die Studenten die Funktionsweise und die Konstruktion der Maschinen zum Fügen von biegeweichen Materialien. Während die Nicht-eingeweihten der Konfektionstechnik nur das Nähen von billigen T-Shirts verstehen, wissen die Fachleute, dass die wissenschaftliche Aufbereitung dieses Faches ein multidisziplinäres tieferes Wissen aus mehreren Fächern erfordert. Die Optimierung eines Nähprozesses für die Verbindung von Bauteilen aus Hochleistungsfasern, sowie das automatische Nähen menschlicher Haut fängt mit der kinematischen Analyse von Getrieben mit komplexen räumlichen Bewegungen an. Die Bewegungsgesetze sind dann nur der erste Schritt für die Analyse der Interaktion zwischen den Maschinenelementen und dem bewegten viskoelastischen eindimensionalen Material - der Nähfaden, der 100 mal in der Sekunde gezogen, dann gebogen, geknickt, durch Reibung erhitzt und am Ende wieder entspannt wird.

Auch komplex ist die Gestaltung der textilen Montage - die biegeweichen Stoffe müssen für einzelne Prozesse an den richtigen Stellen gefaltet werden, dann als mehrere Lagen an der Maschine zusammen geführt und danach umgefaltet, aufgedreht, thermofixiert usw. werden. Bevor ein Ingenieur so einen Prozess zu robotisieren oder zumindest teilweise zu automatisieren anfängt, müssen erstmal diese Schritte vollständig verstanden sein. In den vor-Covid Zeiten im Präsenz-Betrieb wurden diese Fähigkeiten als praktische Übungen direkt an den Näh- und Schweißmaschinen und den Bügelautomaten beigebracht. Wie schon Aristoteles wusste [2] und die Pädagogen bestätigen - die Wahrscheinlichkeit des Behaltens beim Selbermachen liegt bei $90 \%$, weil hierbei gleichzeitig mehrere Kanäle - Sehen, Hören, Tasten angesprochen werden.

Diese Arbeit stellt einige Probleme vor, die bei der Vorbereitung von einzelnen Inhalten für das Distanz-Selbstlernen, welches nötig war um die Präsenzzeiten und die Anzahl der Personen in den Laboren im Rahmen der zulässigen Begrenzungen zu halten, aufgetreten sind.

\section{Typische Aufgaben}

Eine der Aufgaben der Montage ist das erlenen der Fadenüberkreuzungen (Stichtypen). Neben dem meist bekannte Doppelsteppstich, der auf Haushaltsnähmaschinen läuft und auch für Verbindungsnähte in der Industrie überwiegend eingesetzt wird, gibt es noch zahlreiche komplexere Stichtypen, unterteilt in mehreren Klassen. In Abb. 1 ist z.B. ein Überdeckkettenstich, so wie in den üblichen Büchern, dargestellt.

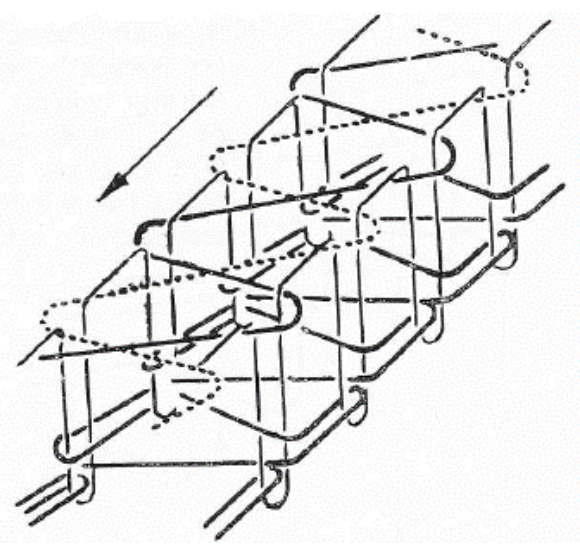

Abb. 1: Überdeckkettenstich [1]

Sitzt man auf der Nähmaschine und erzeugt an dieser selber diesen Stichtyp, sind die Bewegungen der einzelnen Organe klar und auch das Endprodukt von oben und unten sichtbar. Der gleiche Effekt ist schwer durch eine Zeichnung zu erzielen.

Die Hauptaufgabe des Praktikums ist das Erlernen des Zusammennähens eines ganzen Produktes wie z.B. Hemd, Laptop Tasche, Sitzbezug oder ähnliches. Bei dieser Aufgabe muss eine längere Reihenfolge von räumlichen Bewegungen beider Hände mit mindestens je 3 deren Finger und deren Zusammenspiel mit Stoffen und Maschinenerfolgen. Dies wird im Präsenzbetrieb durch "zeigen" und "wiederholen lassen" realisiert.

\section{Basis für die Implementierung Doku- wiki - das "Lernstudio"}

OPAL ist ein mächtiges Tool für Regelung des Zugangs zu einem Stoff und Datenspeicher. Die eingebauten Wikis und Lernmodule waren zu Beginn der COVID-Zeit nicht für serielle Bearbeitung von großen Mengen von Bildern und 
Materialien geeignet. Um auf das Semester "Lehre ohne Hörsaal" immer noch zeitlich gut vorbereitet zu sein, kam nach mehrwöchigen Kampf mit Opal die spontane Entscheidung des Autors, auf dem Webspace der Professur, welche als ein DokuWiki [3] läuft, ein zweites Wiki zu installieren und dort die Inhalte zu gestalten. Somit wurde das "Lernstudio der Professur Entwicklung und Montage von textilen Produkten" geboren.

Warum Dokuwiki als Wiki? Die Entscheidung wurde schon vor Jahren, auf Basis der Wikimatrix [4] und Tests mit MediaWiki, TWiki, PhP Wiki, WordPress und Joomla intern getroffen.

Gründe dafür:

- es ist frei-zugänglich und open source

- es ist klein und fein (3,6 MB Installation)

- es braucht nur PHP und keine SQL-Datenbank, somit läuft es auf dem Webspace der TUD

- es nutzt reine Textdateien für die Beiträge, auf die auch im Falle eines theoretischen Absturzes weiter direkt zugegriffen werden kann und die dann noch verwendbar sind

- es liefert ein einfaches Backup aller Daten

- eine einfache Wiederherstellung durch Entpacken des Backups aus dem Ordner ist möglich

- es hat Namespaces (Ordner) mit der Möglichkeit Zugriffsrechte zu regeln

- es erlaubt das Einbetten von YouTube Videos

- es hat eine einfach zu erlernende Syntax

- es sind mehrere Plug-Ins vorhanden

- HTML, Latex auch PowerPoint (durch VBA Script) lassen sich durch pandoc [5] in Dokuwiki Format konvertieren

- Der Autor pflegt schon mehrere solcher Wikis und hat Erfahrungen damit. Somit ist die Erstellung von Unterrichtsmaterialien schnell realisierbar.

\section{Stichtypen virtuell darstellen}

Um das aktive Verstehen des Lernmaterials: Überkreuzung der Nähfäden, nach dem Prinzip "selber machen", zu ermöglichen wurde die folgende Aufgabe konzipiert: Jeder Student bekommt einen individuellen Sticharttyp, zudem soll ein parametrisches 3D Model in Python erstellt werden. Für die 3D Datensatz-Visualisierung wird vom Autor ein frei zugänglicher Viewer zur Verfügung gestellt. Zum Abschluss der Aufgabe sollen die Fadenlängen berechnet werden. Bei ca. 15 Studenten gab es keine Probleme die individualisierten Aufgaben zu verteilen, nach der ersten ZOOM-Unterrichtseinheit zu diesem Thema zeigte sich jedoch:

Lesson Learned 1: Die Studenten im 8. Semester haben zwar "schon mal" programmiert (und die Informatik Prüfung in C\# bestanden), aber richtig algorithmisches Denken können sie nicht aufweisen.

Lesson Learned 2: Von den Programmierungseinheiten, im ersten Semester, ist nicht viel Wissen behalten worden, da scheinbar kaum jemand der verehrten Kollegen danach fragt oder es pflegt.

Als Ergebnis wurden die folgenden Inhalte als "Schritt für Schritt Einleitung - Python für Beginners" (Abb.2), "Naht-Bild als 3D Projektion, die Koordinaten werden parametrisch beschrieben und in Python kodiert" im Lernstudio vorbereitet. Es wurde eine einfach zu installierende und "pflegeleichte" Umgebung "Thonny" [6] gefunden.

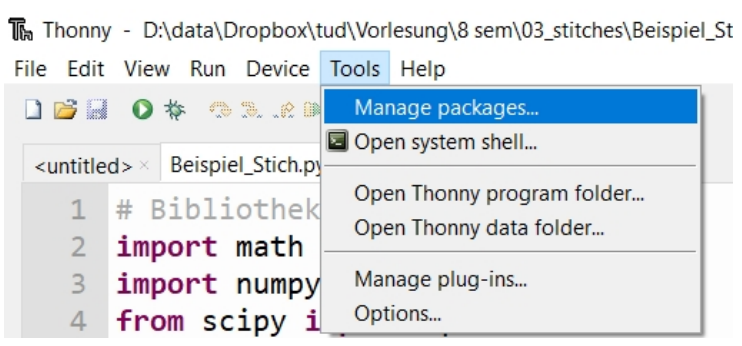

Abb. 2: Bild aus der Schritt für Schritt Anleitung für Thonny

Nach zwei Einheiten je 90 Minuten und einer zusätzlichen Demonstration der Schritt für Schritt Anleitung, kam der "Aha Effekt" bei den Studenten mit den ersten bunten Bildern als Ergebnis, wie in Abb. 3.

Der Versuch die Studenten allein die Länge des Fadens bestimmen zu lassen, scheiterte. Hierbei haben die Hinweise über numerische Integration oder den Pythagoras im Raum und "Schleife" nicht wirklich geholfen. In weiteren Zoom-Meetings wurde die Algorithmik abgehandelt, wie man mit einer Schleife durch die 
Koordinaten geht und wie Abstände addiert werden.

Lesson Learned 3: Die mit "Handy in der Tasche" geborene Generation kann immer noch nicht das Gelernte in Mathematik, Numerische Verfahren und Informatik praktisch anwenden.

Die meisten Studenten fanden die Wiki Inhalte mit Bildern und ausführlichen Erklärungen (es ist ein Elektronisches Handbuch) angenehmer zu nutzen als aufgenommene Videos, weil sie die Inhalte mit ihrer eigenen Geschwindigkeit durchgehen konnten.

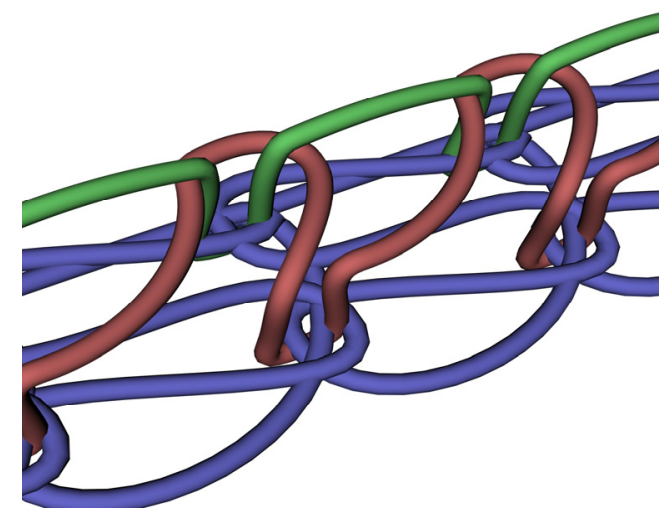

Abb. 3: Generiertes 3D Bild auf Basis eines parametrischen Models des Studenten K.H.

Lesson Learned 4: Text mit Erklärungen ist besser zu folgen, die kleine Datenmenge liegt bei einer Größe ca. 2-3 MB pro Unterrichtseinheit.

Lesson Learned 5: Wiederaufnahme der Vorlesung wird trotzdem gewünscht.

Trotzdem haben einige Studenten gebeten, die aufgenommenen Inhalte mit Erklärungen über ZOOM zusätzlich zur Verfügung zu stellen. (Datenmenge $\mathrm{x} 00 \mathrm{MB}$ pro Unterrichtseinheit)

\section{Montagetechnologie}

Die Technologie der Montage von einzelnen Produkten in der Industrie wird für 4 gelernte "Operatoren von Nähmaschinen" als Tabelle mit Maschinentypen und kurzen Operationsbeschreibungen geliefert, natürlich ergänzt mit Bildmaterial, wie in Abb. 4 dargestellt. Dort werden die Querschnitte an den Verbindungsstellen und die dazu gehörigen Nähte dargestellt.
Das Bild in Abb. 4 zeigt zwar den "fertigen Zustand" der Verbindungsstellen, aber nicht die Reihenfolge der Hand oder Maschinenbewegungen, mit denen die weichen Textilien gebogen, geführt und genäht werden. Auf Basis dieses Bilds per ZOOM mehr zu erklären ist sinnlos.

Eine Alternative, was viele Leute begeistert, sind die Videos auf YouTube (Abb. 5). Praktisch zu realisieren bedeutet es, dass man sich zu Hause eine Nähmaschine beschafft oder ausleiht, den Rechner daneben stellt und sich jeden einzelnen Schritt anschaut, das Video unterbricht und es dann selber praktisch wiederholt.

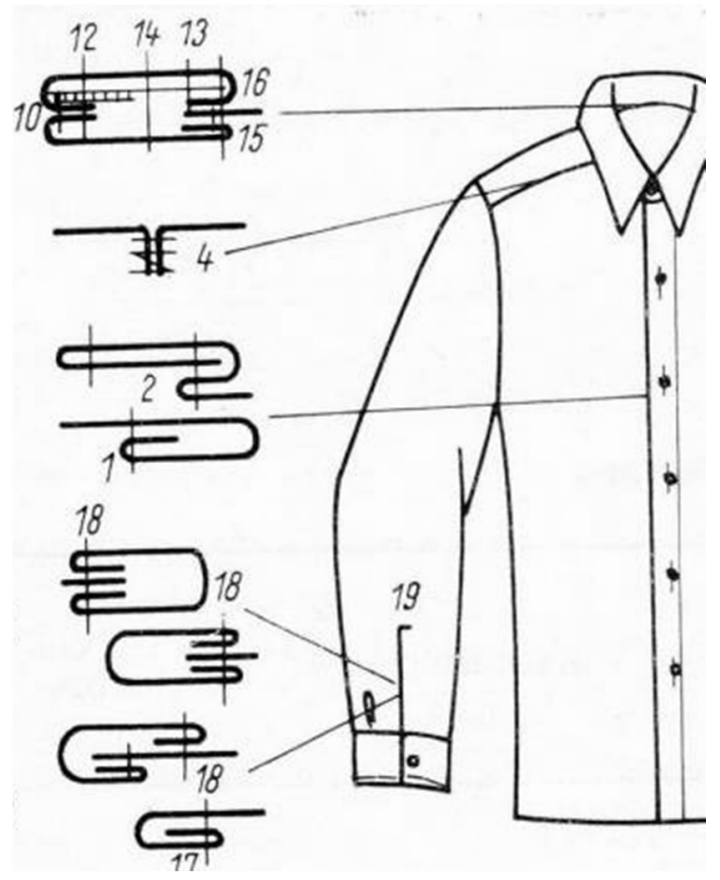

Abb. 4: Auszug aus der Reihenfolge für die Montage eines Hemdes [1]

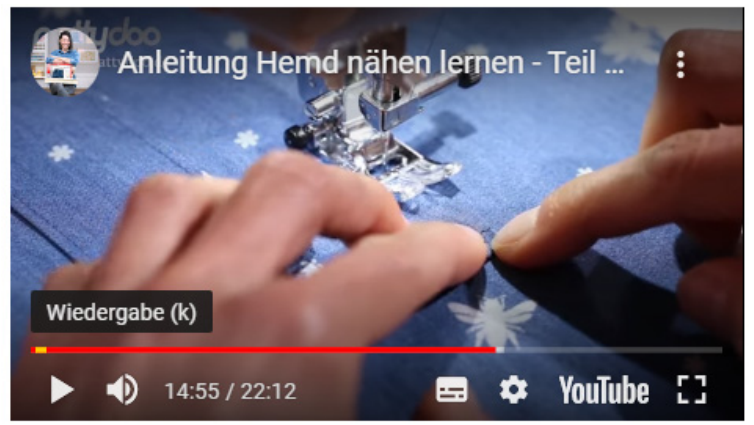

Abb 5. YouTube Video zum Erstellen einer Tasche https://www.youtube.com/watch?v=Zx4yKzfRrus 
Prinzipiell zeigt sich dies als eine gute Lösung, wenn man die lästige Werbung in YouTube überwinden möchte. Durch die Nutzung des neu gestalteten Video-Campus Sachsen [7] ist dieses Problem gelöst und zusätzlich sind die Autorenrechte und der Zugang sehr professionell geregelt.

Einige Nachteile haben die Videos als Medium trotzdem - man kann sich nicht das ganze im schnellen Durchgang übersichtlich anschauen und sich dann auf gezielte Stellen konzentrieren und sie erfordern trotzdem viel Datenvolumen, gute Internet-Verbindung und viel Vorbereitungsaufwand im Aufnahme und Videoschnitt.

Aus diesem Grund wurde nach alternativen Varianten gesucht, wie z.B. bildliche Darstellung der einzelnen Operationen, mit etwas Text, wie sich es in ein klassisches Buch gehört (Abb. 6).

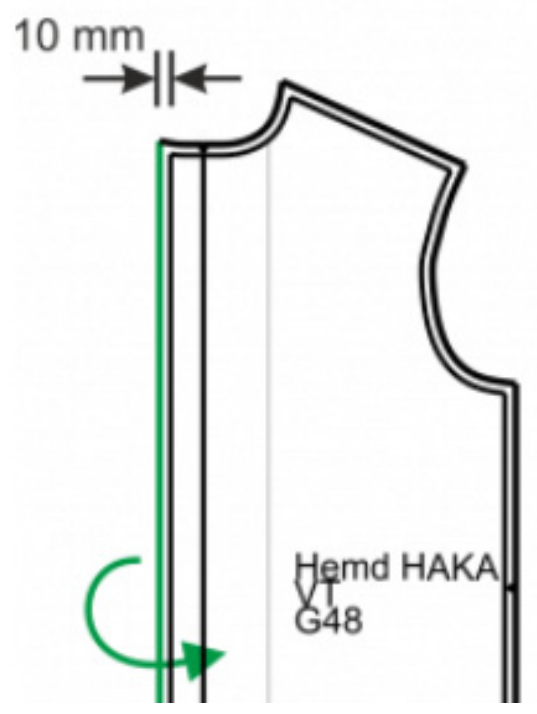

Abb 6. Darstellung einer Operation bei der Fertigung

Diese Variante erforderte deutlich weniger Speicherplatz, ist etwas klarer als einzelne Querschnittsbilder und ermöglicht schnelle Durchsicht und Vertiefung an einzelnen Stellen. Vom Aufwand her ist diese Variante nicht schneller als die Fertigung eines Videos - hier musste auch ein Mitarbeiter einige Tagen mit CorelDraw die Diagramme bearbeiten.

Die Testphase mit ca. 15 Studenten im ersten Durchlauf hat diese Darstellung als nicht so richtig hilfreich gefunden. Bei der Rückfrage über die Gründe wurde erläutert, dass das
Problem nicht bei der Darstellung sondern an der Anwendung lag - hier wurden die Studenten gebeten, sich die Reihenfolge anzuschauen und zu Hause "trocken" zu üben (was natürlich nicht geschah), um danach während des Praktikums mit weniger Erklärungen vorwärts zu kommen. Wie führende Augmented Reality Anwender müsste man hier - ob YouTube Video oder Bilder als Bildserie - ein AR Glass oder zumindest ein Tablet oder Laptop neben die Nähmaschinen stellen damit es einen Effekt hat.

An diese Möglichkeit wurde auch gedacht, allerdings stellte sich das Problem heraus, dass:

a) aus Platzgründen kein Laptop gerade neben die Maschine passt

b) es technologisch sinnvoll ist, dass der Student sein Tablet/Screen/Laptop mit sich führt und die nächsten Schritte ansieht.

Diese letzte Option ist in Planung und wird in der näheren Zukunft getestet, weil es auch in normalen Zeiten eine dynamischere Gestaltung der Praktika ermöglicht, wo jeder Student mit seiner eigenen Geschwindigkeit die Aufgaben bearbeiten kann.

\section{Zusammenfassung}

Die Einführung der Programmierung von 3DNähten hat nur teilweise die praktische Übung ersetzt, hat aber auch dazu geführt, dass die Studenten Methoden für Darstellung der Nähte gelernt haben und diese Darstellungen später direkt für FEM Berechnung verwenden können. Diese Einheit wird auch in nach-Covid Zeiten fortgesetzt.

Die Darstellung der Technologie der Fertigung als Video oder Reihe von Bildern mit Erklärungen ist möglich, allerdings erfordert deren Anwendung dass diese Inhalte auf einem tragbaren Tablett direkt neben der Maschine mitgenommen werden. Diese Implementierung wird auch in der Zukunft getestet und weiterentwickelt um den Betreuungsaufwand zu optimieren und mehr Individualisierung bei den praktischen Einheiten zu schaffen.

Mails wie diese " ich habe viel gelernt und fand die Aufgaben super, hat mir Spaß gemacht. :)" (A.G.,13.08.2020) haben meine schon mehr als 20-jährige Lehrerfahrung bestätigt, dass der 
Weg ein mit individuellen Aufgaben begleitendes Semester zu gestalten, und diese prüfungsrelevant zu honorieren - richtig gewesen ist.

\section{Danksagung}

Herzlichen Dank an Frau Claudia Neumann SG 7.5 und dem Websupport Team für die kompetente Beratung und schnelle Regelung des Webspaces der Professur, wo das Lernstudio implementiert wurde.

\section{Literatur}

[1] Endre, N., Ferenc, T., Ruhaipari kezekönyv, MuszakKönyvkiado, Budaperst, 1979

[2] Knoll, Michael, Vom Aristoteles zu Dewey. Zum Usprung der Maxime "learning by doing", https://www.mi-knoll.de/128401.html

[3] www.dokuwiki.org

[4] www.wikimatrix.org

[5] www.pandoc.org

[6] www.thonny.org

[7] https://videocampus.sachsen.de/ 